\title{
Pneumococcal vaccination process improvement in an acute care setting
}

\author{
Abigail M Yancey, ${ }^{1}$ Anne B Jundt, ${ }^{2}$ Kathryn J Nelson ${ }^{2}$
}

1Department of Pharmacy Practice, St Louis College of Pharmacy, St Louis, Missouri, USA

${ }^{2}$ Quality Management Department, SSM St Mary's Health Center, St Louis, Missouri, USA

\section{Correspondence to} Dr Abigail M Yancey, Department of Pharmacy Practice, St Louis College of Pharmacy, 4588 Parkview Place, St Louis, M0 63110-1088, USA; ayancey@stlcop.edu

Accepted 21 October 2008 Published Online First 25 August 2010

\begin{abstract}
Background and objective Despite the availability of the pneumococcal vaccine since 1977, the vaccine is greatly underutilised. Centers for Medicare and Medicaid Services, The Joint Commission and Healthy People 2010 have all listed the administration of the pneumococcal vaccine before hospital discharge as a standard of care and a quality initiative in the 21st century. SSM St Mary's Health Center chartered a multidisciplinary team to address a disappointing pneumococcal vaccination rate of $34.7 \%$ in the first quarter of 2005.
\end{abstract}

Methods The team utilised the improvement model of Plan-Do-Study-Act to implement and monitor process changes. Changes were made to four key steps in the pneumococcal vaccination process: assessment, ordering, obtaining and administering. The team also implemented a concurrent review process. The team tracked the hospital's pneumococcal vaccination rate per the published Centers for Medicare and Medicaid Services and The Joint Commission guidelines.

Results Over a 2-year period, the vaccination rate of pneumonia patients has improved incrementally from $34.7 \%$ and is now consistently greater than $90 \%$. Conclusion Utilising Plan-Do-Study-Act allows for continual improvement of the vaccination process. Multiple cycles are necessary to achieve standardisation and optimal process flow.

In 2001, the Department of Health and Human Services announced their plan for a national quality initiative. The goal of this initiative was to 'assure quality healthcare for all Americans through published consumer information." ${ }^{1}$ To achieve this goal, Centers for Medicare and Medicaid Services (CMS), a division of Health and Human Services, collaborated with The Joint Commission to standardise the collection and reporting of hospital quality performance data. Initially, four core measurement areas for hospitals were established: pneumonia, acute myocardial infarction, heart failure, and pregnancy and related conditions. In late 2004, SSM St Mary's Health Center (SMHC) voluntarily joined the national quality initiative. SMHC, a 582-bed community teaching hospital, serves the city of St Louis, Missouri. As an organisation focused on quality, SMHC senior leadership charted multidisciplinary improvement teams focused on the core measures. The pneumonia team consisted of healthcare professionals from Emergency Medicine, Nursing, Pharmacy, Respiratory Therapy, Staff Education, Internal Medicine, Case Management and Quality Management. The goal was to improve care provided to patients admitted with the diagnosis of pneumonia.

\section{OUTLINE OF PROBLEM AND CONTEXT}

The pneumococcal vaccination rate was among the initial data reviewed by the team. The pneumococcal vaccination rate, as defined by The Joint Commission and CMS, is the number of "pneumonia patients, age 65 and older, who were screened for pneumococcal vaccine status and were administered the vaccine prior to discharge, if indicated. ${ }^{1}$ This is an important performance measure for SMHC because $50 \%$ of patients admitted are 65 years and older. Initial data in the fourth quarter 2004 showed that the SMHC pneumococcal vaccination rate was at $52 \%$, just below the CMS national average. However, by first quarter 2005, SMHC had a disappointing rate of $34.7 \%$ for patients discharged with the diagnosis of pneumonia.

The 23-valent polysaccharide pneumococcal vaccine has been available since 1977 and is recommended for all patients 65 years of age or older and for younger individuals with certain medical conditions and risk factors. ${ }^{23}$ Despite the availability, the vaccine is still greatly underutilised. According to the Centers for Disease Control and Prevention, the national pneumococcal vaccination rate is less than $60 \%$ for the 65 years of age and older population. ${ }^{4}$ In 2007 , the combined death category of pneumonia and flu was listed as the seventh leading cause of death for people over the age of $65 . .^{5}$ More than 900000 cases of pneumonia occur each year among seniors in the USA, and 600000 of those were hospitalised. ${ }^{6}$ Of the patients hospitalised with serious pneumococcal infections, up to two-thirds had been hospitalised within the previous 3-5 years. ${ }^{8}$ Thus, CMS, The Joint Commission and Health People 2010 have all listed the administration of the pneumococcal vaccine before hospital discharge as a standard of care and as a quality initiative in the 21 st century. ${ }^{19}$

\section{ASSESSMENT OF PROBLEM}

With the unacceptable vaccination rate of $34.7 \%$, the team began to address the rate utilising the improvement process model of Plan-Do-Study-Act, also known as PDSA. ${ }^{10}$ The initial PDSA cycle began by evaluating the current vaccination process. Evaluation identified that there were no set processes in place for assessment and administration of the pneumococcal vaccine. Assessment of and ordering of the vaccine was at the discretion of each physician. However, physicians do not routinely order vaccines for patients in the acute care setting or document their previous vaccination history. To address the problem, the team set out to design, trial and establish a formalised process to optimise vaccination rates. 


\section{STRATEGIES FOR CHANGE}

The following discussion outlines the iterations of PDSA that took place over approximately 2 years. There are four key steps in the process that will be addressed: patient assessment, ordering, obtaining and administering of the vaccine, and feedback through concurrent review.

\section{Patient assessment}

The team designed a detailed nurse-initiated assessment form that included all potential indications for pneumococcal vaccinations and required a physician signature upon completion (figure 1). The form was placed in the hospital admission packet, but there was no mandatory process designed for using the form. Assessment was at the discretion of the nurses. Feedback from nursing regarding the form was less than positive, and barriers were identified. The nurses were not completing the forms because they were cumbersome and time-intensive, and the population needing to be assessed was unclear. Physicians were also not signing the assessment form, thus never making it an order.

To address the barriers, the team redesigned the assessment form to focus on age greater than or equal to 65 as the primary risk factor. This change allowed for a condensed form that included pneumococcal and flu assessments on a single sheet.

\begin{tabular}{|c|c|}
\hline \multicolumn{2}{|c|}{ PNEUMOCCAL VACCINATION Patient Assessed by:____ Date: } \\
\hline $\begin{array}{l}\text { Step } 1 \\
\text { ASSESS FOR CONIRAINDICATIONS: Vaccine NOT indicated } \\
\square \text { Pregnant } \\
\square \text { Previous y immunized after age } 65 \\
\square \text { Previously immunized before age } 65 \text {, but less than } 5 \text { years ago } \\
\square \text { Reported allergy to vaccine } \\
\square \text { VACGNE CONTRAINDICATED, STOP ASSESSMENT. PLAC } \\
\text { IF PATIENT HAS NO CONTRANDCATIONS AND HAS NOT REC } \\
\text { HISTORY, PROCEED TO STEP2** }\end{array}$ & $\begin{array}{l}\text { due to (check box that applies) } \\
\square \text { Patient refuses } \\
\square \text { Patient has already received initial and one time } \\
\text { revaccination Date: } \\
\text { CEFORM IN ADMISSIONDATA SECTIONOF THE CHART. } \\
\text { CEVED PNEUMONA VACCIANTIONOR HAS UNKNOWN }\end{array}$ \\
\hline $\begin{array}{l}\text { Step } 2 \\
\text { ASSESSFOR RISKFACTORS: (Patient appropriate candidate fo } \\
\text { Check all that apply: } \\
\square \text { 65years of age or older } \\
\square \text { Resident of nursing home or long term care fadility } \\
\square \text { Alaska native or Native American } \\
\square \text { Patient } 19 \text { years of age or older with any of the following: } \\
\square \text { chronic heart disease } \\
\square \text { diabetes mellitus } \\
\square \text { chronic liver disease } \\
\square \text { functional/anatomic asplenia (includes sickle œll disease) } \\
\square \text { chemotherapy with alkylating agents or antimetabolites } \\
\square \text { immunocompromised (congenital immunodefidiency, Hodgkir } \\
\text { malignancy, HIVIAIDs, organ transplant) } \\
\square \text { NORISK FACTORS ARE IDENTIFED, STOP ASSESSMENT. P } \\
\text { IF RISK FACTORSPRESENT, PROCEFD TO STEP3 ORTOSTE }\end{array}$ & $\begin{array}{l}\text { or vaccinationif one ormore risk factors of identified) } \\
\square \text { chronic lung disease (excludes asthma) } \\
\square \text { chronic renal failure or nephrotic syndrome } \\
\square \text { CSF leaks } \\
\square \text { long-term corticosteroids } \\
\text { in's disease, leukemia, lymphoma, multiple myeloma, generalized } \\
\text { PLACE FORMIN ADMISSION DATA SECTION OF THECHART. } \\
\text { EP 4*k }\end{array}$ \\
\hline 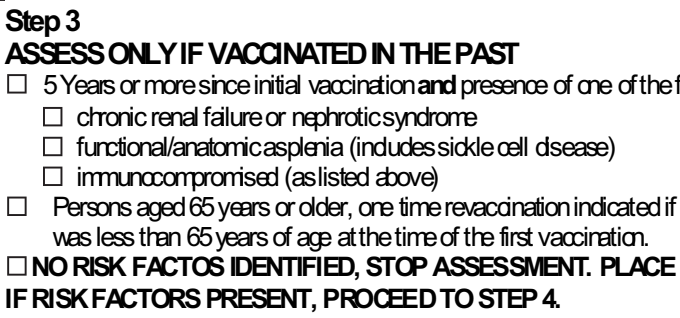 & $\begin{array}{l}\text { fdlowing conditions: } \\
\qquad \begin{array}{l}\quad \text { demotherapy (as listed above) } \\
\square \text { long-termcorticosteroids }\end{array} \\
\text { fit hæs been } 5 \text { years or more sinœ the first vaocindion and the patient } \\
\text { FORM IN ADMISSION DATA SECTION OF CHART. }\end{array}$ \\
\hline $\begin{array}{l}\text { Step } 4 \\
\square \text { Patient Identified for Risk Factor(s) (Obtain Consent) } \\
\square \text { Unknown Vacination History (Obtain Consent) } \\
\text { **IF PATIENT HAS RISK FACTORS, IS WTHOUT CONTRAINDIC } \\
\text { PNEUMOCOCCAL VACCINE 0.5m IM ON DAY OF DISCHARGE }\end{array}$ & $\begin{array}{l}\text { CATIONS, AND ISEUGIBLE FOR VACCINATION, GNE } \\
\text { E, UNLESS BELOWCONSIDERATIONS ARE PRESENT.** }\end{array}$ \\
\hline $\begin{array}{l}\text { CONSIDRATIONS } \\
\text { - If patient currently receiving chemotherapy, has severe neutroperia; has a } \\
\text { Oncology/Hematology or PCP before ordering immurization } \\
\text { - If patient has autaimmune dsease (Autaimmune thrombocytopenial hemolyt } \\
\text { - Iumira, Kineret), pleasecheck with Rheumat dogy or PCP beforeordering } \\
\text { - If patient has active multiple sclerosis, please check with Neurdogy or PCP }\end{array}$ & $\begin{array}{l}\text { known bleeding disorder or platelet count less than 50; please check with } \\
\text { tic anemia; systemic lupus); or is receiving a bidogicagent (Remicade; Enbrel, } \\
\text { immunization } \\
\text { before ordering immunization }\end{array}$ \\
\hline $\begin{array}{l}\text { Step } 5 \\
{ }^{*} \text { STANDINGORDER: MAY GIVE PRIOR TO PHYSICIA } \\
{ }^{*} \text { SCAN TOPHARMACY** } \\
{ }^{*} \text { PLACE ORDERFORM IN ORDER SECTION OF CHAF }\end{array}$ & WSIGNATURE \\
\hline Physician Signature & Date \\
\hline
\end{tabular}

Figure 1 SSM St Mary's Health Center (SMHC) Original Vaccine Assessment Form. 
Physician signature was no longer required by state law, so it was removed from the form. Positive feedback was received; however, barriers were still identified. Some nurses were still not using the form, since they were not mandatory for all patients. Additionally, the final step of initiating the order was often omitted because after the nurse assessed for indications, they had to mark an additional box to finalise the order (figure 2).

To increase compliance with vaccine assessments, the nurses needed one rule to follow. Thus, the assessment form was made mandatory for all patients admitted to the hospital regardless of age. This change was made possible by making age a criterion for indication and contraindication. The form underwent a final change to a decision-tree format with easy-to-follow arrows (figure 3). The team also outlined on the back side of the assessment sheet other potential indications for the vaccine that would need a physician order.

\section{Ordering}

Since the assessment form was now to be initiated by nursing, the standing order protocols of the hospital needed to be updated to reflect the change. Our medical staff approved the assessment criteria for the vaccine to make it a standing order

\begin{tabular}{|c|}
\hline $\begin{array}{l}\begin{array}{l}\text { Pneumococcal Vaccine: Assess only if patient } 65 \text { years or greater } \\
\text { Influenza Vaccine: Assess only if patient } 50 \text { years or greater }\end{array} \\
\text { VACCINATION(S) Patient Assessed } \\
\text { by: } \\
\text { Standing order: No physician signature required effective } 2 / 13 / 06\end{array}$ \\
\hline PNEUMOCOCCAL VACCINATION \\
\hline $\begin{array}{l}\text { ASSESS FOR CONTRAINDICATIONS: Disqualifying factors (one or more checked boxes) } \\
\square \text { Patient refuses vaccine } \\
\square \text { Reported allergy to vaccine or components } \\
\square \text { Previously immunized after age } 65 \text { Date: } \\
\square \text { Previously immunized before age } 65 \text {, but less than } 5 \text { years ago } \\
\square \text { Physician has ordered NOT to administer vaccine during hospitalization } \\
\square \text { Code Status Level 3: Comfort Measures }\end{array}$ \\
\hline $\begin{array}{l}\text { ASSESS FOR INDICATIONS: Qualifying factors (one or more checked boxes) } \\
\square 65 \text { years of age or older } \\
\square \text { Resident of nursing home or long term care facility } \\
\square \text { Unknown Vaccination History (unsure if or when vaccination was given) } \\
\square \text { Revaccination due if patient received shot before age } 65 \text { and it has been more than } 5 \text { years }\end{array}$ \\
\hline $\begin{array}{l}\text { Pneumovax NOT INDICATED: PLACE FORM IN ADMISSION SECTION OF CHART } \\
\begin{array}{l}\text { Pneumovax INDICATED: } \\
\text { OR PRIOR TO DISCHARGE }\end{array} \\
\begin{array}{ll}\text { 2. SCAN TO PHARMACY } \\
\text { 3. PLACE ORDER FORM IN ORDER SECTION OF CHART }\end{array}\end{array}$ \\
\hline INFLUENZA VACCINATION: ASSESS ONLY OCTOBER THRU MARCH \\
\hline $\begin{array}{l}\text { ASSESS FOR CONTRAINDICATIONS: Disqualifying factors (one or more checked boxes) } \\
\square \text { Patient refuses vaccine } \\
\square \text { History of allergy or anaphylaxis to thimersol (contact lens solution), eggs, or the influenza } \\
\text { vaccine } \\
\square \text { Patient has received vaccine this season (October - March) } \\
\text { IF YES: Month } \\
\square \text { Physician has ordered NOT to administer vaccine during this hospitalization } \\
\square \text { Code Status Level 3: Comfort Measures }\end{array}$ \\
\hline $\begin{array}{l}\text { ASSESS FOR INDICATIONS: Qualifying factors (one or more checked boxes) } \\
\square \text { Patient is hospitalized and is } 50 \text { years or over during October - March } \\
\square \text { Resident of nursing home or long term care facility }\end{array}$ \\
\hline $\begin{array}{l}\text { Influenza vaccine NOT INDICATED: PLACE FORM IN ADMISSION SECTION OF CHART } \\
\square \text { Influenza vaccine INDICATED: } 1 \text {. GIVE INFLUENZA } 0.5 \text { mI IM } 48 \text { HOURS AFTER } \\
\text { ADMISSION OR PRIOR TO DISCHARGE } \\
\text { 2. SCAN TO PHARMACY } \\
\text { 3. PLACE ORDER FORM IN ORDER SECTION OF CHART }\end{array}$ \\
\hline
\end{tabular}

Figure 2 SSM St Mary's Health Center (SMHC) Revised Vaccine Assessment Form. 


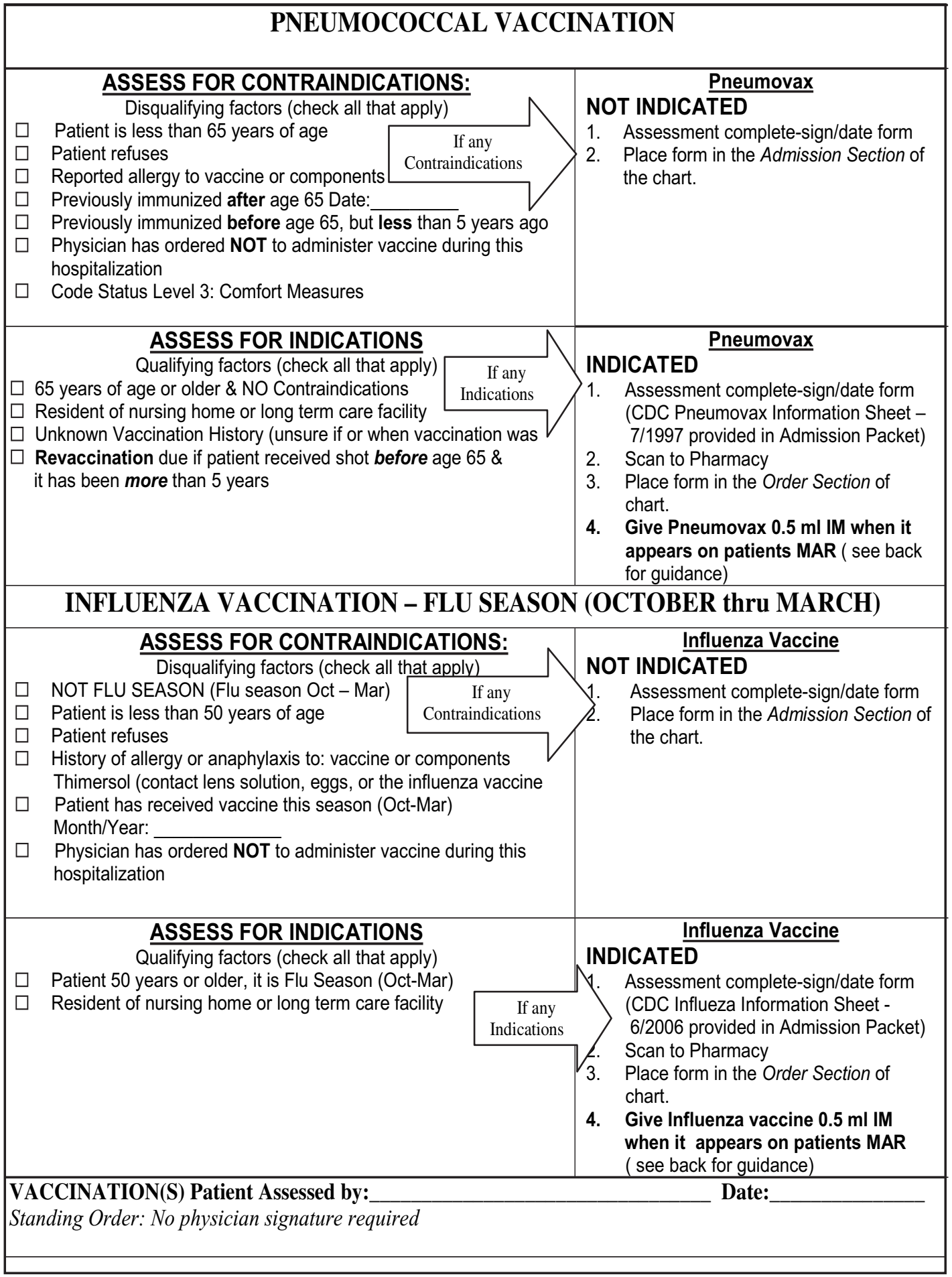

Figure 3 SSM St Mary's Health Center (SMHC) Final Vaccine Assessment Form.

Thus, nursing staff or pharmacy staff could assess patients and administer the vaccine prior to the physician's signature. However, the physician's signature was still required prior to discharge. Nurses were reluctant to vaccinate the patients prior to the physician's signature. Physicians frequently missed signing the assessment sheet; consequently, eligible patients were not vaccinated.

To address the process failure, the requirement for a physician's signature was removed from the assessment form. State legislation did not require a physician's signature for administration of the vaccine. Removal of the signature allowed the process to be owned by nursing and pharmacy staff; however, adoption of this practice was not an easy transition. Implementation of a concurrent review process was identified as a need in this transition. (A concurrent review process is described in a separate section.) This review would allow for intervention at the point of care and education to staff on the safety, efficacy and importance of the vaccine. This new process was facilitated through a pharmacist concurrent reviewer of pneumonia patients. 


\section{Obtaining and administering}

The initial process for obtaining and administering the vaccine began once the completed assessment form had been signed by the physician. The unit secretary would then scan the form to pharmacy. Once the order was received, the pharmacist would enter the vaccine on the patient profile as a demand medication. Per hospital policy, demand medications are not sent up to the nursing unit until requested. As a daily reminder to nursing, the vaccine order was displayed on the patient's medication administration record throughout their stay. On the day of discharge, the nurse was responsible for contacting the pharmacy to have the dose delivered. When contacted, the pharmacist would send the nurse a written consent form for the patient to complete. Once the consent form was completed and sent to the pharmacy, the pharmacist would prepare the vaccine and deliver it to the appropriate medication station. The nurse would then pull the vaccine from the medication station and administer the vaccine to the patient.

This initial process involved many steps, differing from typical nursing and pharmacy medication practices. Nursing identified that once discharged, patients were reluctant to wait for the vaccine to be prepared and delivered by the pharmacy. The demand process took too long; therefore, patients were discharged without being vaccinated. To shorten this wait time, the team first began process redesign by removing the written consent at administration of the vaccine. SMHC approved and implemented verbal consent for administration of the vaccine in accordance with state law. The pharmacy suggested storage of the vaccine on the nursing units in the medication administration stations, which reflects current hospital medication practices. This change eliminated the time-intensive steps of the nurse calling pharmacy and pharmacy preparing and delivering the dose.

Although the process was streamlined, vaccines were still being missed. Two additional barriers were identified. The first was that the vaccine was continually on the patient's medication administration record with no set date for administration, causing it to be ignored or overlooked. The second barrier was administering the vaccine at discharge, which was not optimal given the hectic nature associated with discharge. Our medical staff approved the team's recommendation to move the scheduled time of administration to day 2 of the hospital stay at 09:00. This change standardised and added certainty to the time and date of administration. The concurrent reviewer could then track vaccination administration and intervene when necessary.

\section{Concurrent review process}

Prior to the implementation of the concurrent review process, pneumonia care was assessed after the patient has been discharged. A concurrent reviewer allows for continual assessment and optimisation of care delivered to pneumonia patients.

Identification of pneumonia patients or case finding is the difficult piece in this process. The team developed a list of common International Classification of Diseases, Ninth Revision (ICD-9) codes associated with pneumonia and pneumonia type symptoms for the reviewer to track (table 1). ${ }^{11}$ The code list was developed based on the most common admission diagnoses associated with patients who had a final pneumonia diagnosis. In addition to tracking the codes, the reviewer follows all patients started on the pneumonia antibiotic protocol. The expectation upon identification of patients with pneumonia is to review care given, intervene and educate staff as necessary.
Table 1 Admitting diagnoses used for case finding in concurrent review

\begin{tabular}{ll}
\hline ICD-9 Code & Definition \\
\hline $038-038.9$ & Septicaemia \\
$480.0-487.0$ & Pneumonia and flu \\
496 & Chronic airway obstruction, not classified \\
& elsewhere \\
$518-518.89$ & Other lung diseases \\
780.6 & Fever \\
$786-786.9$ & Symptoms involving respiratory system \\
& and other chest symptoms \\
\hline
\end{tabular}

\section{EFFECTS OF CHANGE}

Rate compliance encompasses two efforts (1) administering the vaccine when indicated or (2) documenting a previous vaccine history. Cumulative pneumococcal vaccination rates were collected from October 2004 to December 2007. These rates were reviewed on a monthly basis in accordance with PDSA. Vaccination rates for patients with a diagnosis of pneumonia increased incrementally on a quarterly basis (figure 4). From the first quarter of 2005 to the third quarter of 2006, vaccine rates increased from $35 \%$ to $92 \%$. Since the third quarter of 2006 , with the exception of the second quarter of 2007, compliance with vaccination has been consistently $90 \%$ or above.

While the results in figure 4 focus on patients with a diagnosis of pneumonia, the hospital identified a substantial increase in overall administration of pneumococcal vaccines (figure 5). Between 2004 and 2007, an additional 1052 doses were dispensed, an astonishing $683 \%$ increase. The bulk of the vaccines dispensed for each year, approximately $40 \%$, occurred in the fourth quarter, which coincides with the beginning of the flu season.

\section{DISCUSSION}

With the increasing costs of healthcare, vaccination can be instrumental in decreasing the cost, morbidity and mortality associated with pneumonia. Thus, the SMHC pneumonia team set out design a process to achieve $100 \%$ compliance with the national standard.

In Don Berwick's central law of improvement, 'Every system is perfectly designed to achieve the results it achieves. ${ }^{12}$ SMHC had no formalised pneumococcal vaccination process. The team worked through multiple improvement cycles identifying successes and barriers. Although multiple cycles were required, the team did not view previous work as a failure. The team learnt that the theoretical processes developed do not always work in the reality of the hospital setting. Assessment of reality, although painful at times, provides the barriers for a potentially successful process. The barriers are necessary to launch subsequent cycles of change.

The current process has allowed achievement as high as $92 \%$. Below outlines, the key lessons in our process improvement journey are as follows:

1. Develop a streamlined process: the key areas to this success include the form redesign, a standing order policy, vaccination on day 2 of stay and a vaccination process that reflects typical medication practices.

2. Education and training: engage nursing staff by educating them on the purpose of the national quality initiative and the evidence to support the measures. Provide nursing with ongoing feedback of performance results both overall compliance and unit specific data. Use the concurrent review process to educate in real time.

3. Real-time intervention: implement a concurrent review process whereby the reviewer follows the patient through 


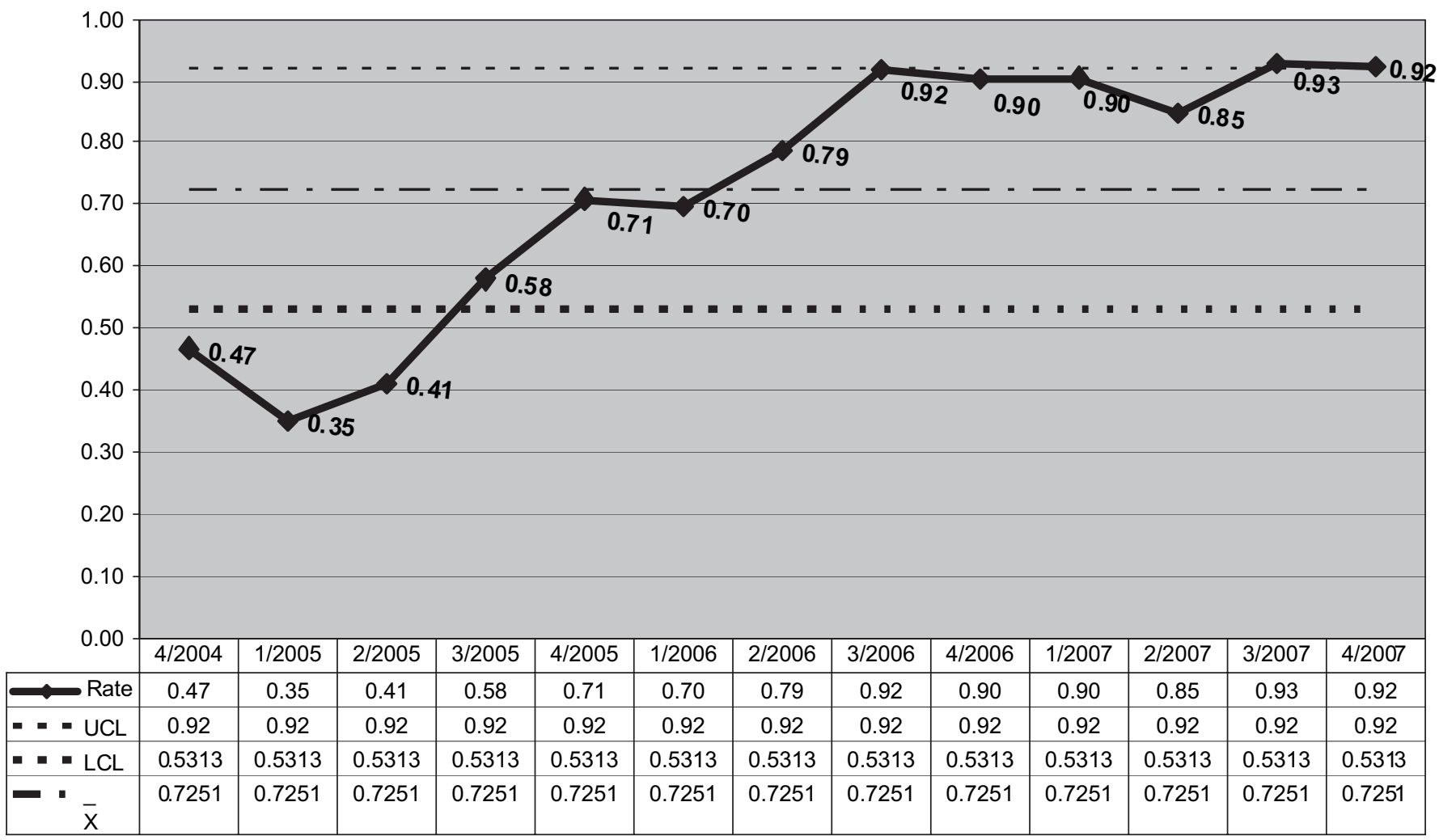

Figure 4 SSM St Mary's Health Center (SMHC) pneumococcal vaccination rates for pneumonia patients I-MR chart of rate. I-MR, (Individuals and Moving Range) of rate.

the process and is able to intervene when necessary to complete the process. The concurrent reviewer will intervene less as the process improves.

4. Multidisciplinary team.

Remaining barriers include a short length of stay for pneumonia patients, nursing turnover and the use of a paper assessment tool. Patients with stays shorter than 2 days are frequently discharged prior to vaccination. Nursing turnover and agency nursing staff causes a knowledge gap with hospital policies and procedures leading to inappropriate screening and missing doses. Finally, SMHC currently uses paper charting leading to missing forms in admission packets and missing completed forms at discharge. This sets the process up for failure and also causes inaccurate counts of patients who were vaccinated prior to admission. SMHC will implement the electronic health record in late 2009.

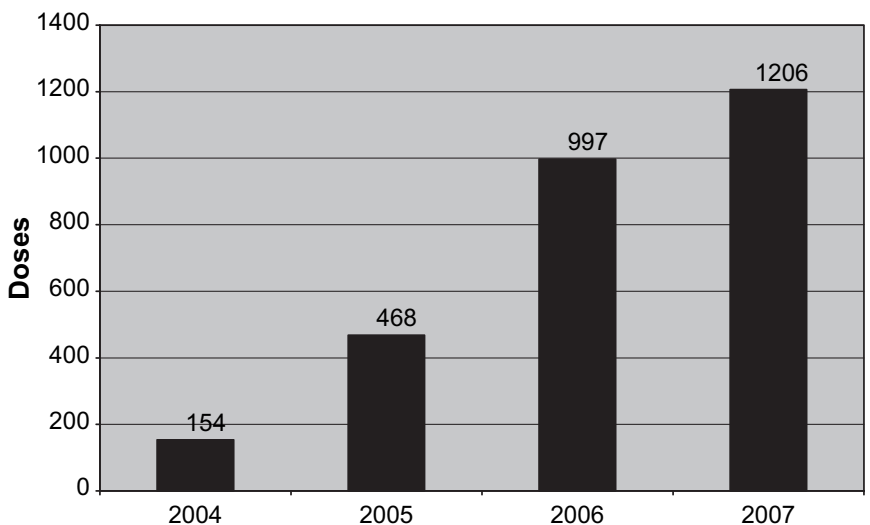

Figure 5 SSM St Mary's Health Center (SMHC) Pneumovax doses dispensed.
Competing interests None.

Provenance and peer review Not commissioned; externally peer reviewed.

\section{REFERENCES}

1. The Joint Commission. Specifications manual for national hospital quality measures 2007. http://www.jointcommission.org/PerformanceMeasurement/

PerformanceMeasurement/Historical +NHOM + manuals.htm (accessed 18 Jan 2008)

2. Mandell LA, Bartlett JG, Dowell SF, et al. Update of practice guidelines for the management of community-acquired pneumonia in immunocompetent adults. Clin Infect Dis 2003;37:1405-33.

3. Lionel AM, Wunderink RG, Anzueto A, et al. Infectious Diseases Society of America/ American Thoracic Society Consensus guidelines on the management of communityacquired pneumonia in adults. Clin Infect Dis 2007;44:S27-72.

4. Centers for Disease Control and Prevention. Public health and aging: influenza vaccination coverage among adults aged $>50$ years and pneumococcal vaccination coverage among adults aged $>65$ years-United States, 2002. MMWR Morb Mortal Wkly Rep 2003;52:987-92.

5. National Center for Health Statistics. Health, United States, 2007, with Chartbook on Trends in the Health of Americans. Hyattsville, MD: National Center for Health Statistics, 2007. http://www.cdc.gov/nchs/data/hus/hus07.pdf (accessed 14 Jan 2008).

6. Jackson ML, Neuzil KM, Thompson WW, et al. The burden of community-acquired pneumonia in seniors: results of a population-based study. Clin Infect Dis 2004;39:1642-50.

7. Kaplan V, Angus DC, Griffin MF, et al. Hospitalized community-acquired pneumonia in the elderly: age- and sex-related patterns of care and outcome in the United States. Am J Respir Crit Care Med 2002;165:766-72.

8. Bratzler DW, Houck PM, Hui J, et al. Failure to vaccinate medicare inpatients-a missed opportunity. Arch Intern Med 2002;162:2349-56.

9. US Department of Health and Human Services. Healthy People 2010. Washington, DC: US Department of Health and Human Services. http://www.healthypeople.gov/Document/html/uih/uih bw/uih 4.htm\#immuniz (accessed 18 Jan 2008).

10. Langley GJ, Nolan KM, Nolan TW et al. The Improvement Guide: A Practical Approach to Enhancing Organizational Performance. San Francisco, CA: Jossey-Bass, 1996:6-11.

11. ICD-9-CM. International Classification of Diseases, 9th Revision, Clinical Modification. 6th edn, 2006. Salt Lake City (UT): Ingenix, 2005:8, 140-2, 144, 146-7, 250, 253

12. Berwick DM. A primer on leading the improvement of systems. BMJ 1996;312:619-22. 